\title{
Projets du Swiss Medical Board en 2012
}

\author{
En 2012, l'organe responsable du Swiss Medical Board prévoit l'évaluation de trois \\ prestations médicales. Il s'agit de l'ablation chirurgicale de métastases hépatiques \\ en cas de cancer de l'intestin et de l'usage de la tomodensitométrie pour le dia- \\ gnostic des maladies coronariennes. Le thème du troisième rapport sera défini ulté- \\ rieurement.
}

Le comité de direction des organes responsables de Swiss Medical Board prévoit l'évaluation de trois prestations médicales en 2012. D'une part le choix des thèmes repose sur des recommandations émanant du corps médical. D'autre part, l'organe responsable envisage cette année d'examiner les possibles synergies avec des rapports des agences HTA à l'étranger. Dans la mesure du possible, deux de ces évaluations devraient pouvoir découler de rapports déjà élaborés et publiés à l'étranger. Le Swiss Medical Board veut s'efforcer de tirer parti des synergies existantes entre les différentes agences HTA; tel est aussi l'objectif de la collaboration internationale dans le cadre du SNHTA (Swiss Network for Health Technology Assessment). L'évidence médicale constitue une part essentielle de tous les rapports HTA. C'est dans cette partie (d'envergure) que l'on espère trouver les principaux effets de synergie. Les aspects juridiques, éthiques et économiques sont spécifiques à chaque pays; ils tiennent compte des environnements respectifs et sont intégrés aux recommandations.

Concrètement, le Swiss Medical Board procèdera cette année aux évaluations suivantes:

\section{Ablation chirurgicale de métastases hépatiques en cas de cancer colorectal}

Le terme de «carcinome colorectal» englobe les cancers du côlon et du rectum. Après l'ablation d'un tel carcinome, 25 à 35\% des patients développent des métastases hépatiques dans les cinq années après l'ablation.

Cette évaluation permettra de vérifier si, par rapport à d'autres procédés thérapeutiques, l'ablation chirurgicale de ces métastases offre au patient concerné un avantage appréciable en terme de survie et de qualité de vie. Ce faisant, les possibles effets négatifs des différents procédés thérapeutiques et la relation coûts-efficacité seront également examinés.

\section{Usage de la tomodensitométrie pour le diagnostic de la maladie coronarienne - une analyse basée sur un rapport HTA de NICE}

La maladie coronarienne (rétrécissement des vaisseaux cardiaques) est une maladie courante qui présente une morbidité et une mortalité élevées. Il existe deux possibilités de diagnostiquer la maladie coronarienne et de mesurer le rétrécissement des vaisseaux: 1 . l'angiographie coronaire invasive: un cathéter est introduit par l'artère inguinale dans les artères coronaires permettant de visualiser les artères et 2. la tomodensitométrie.

Chez un groupe de patients présentant une suspicion de maladie coronarienne, la tomodensitométrie ne permet d'établir qu'un diagnostic peu précis avec les appareils traditionnels. De nouveaux appareils (lesdits «NGCCT, new generation cardiac CT») devraient combler ces lacunes.

Dans cette étude, c'est la précision diagnostique des nouveaux appareils NGCCT qui est examinée. L'angiographie coronarienne sert d'élément de comparaison. Il s'agit également de vérifier si ce procédé offre un avantage au patient. En plus, les possibles effets négatifs des deux procédés et la relation coûtsefficacité seront examinés.

Ce rapport est basé sur une synthèse de l'évidence, élaborée par le National Institute for Health and Clinical Excellence (NICE) britannique.

Le thème d'une troisième évaluation sera défini ultérieurement.

L'approbation et la publication des rapports sont prévues pour la fin de l'année.

Informations complémentaires sur les projets du Swiss Medical Board: www.medical-board.ch/index. php?id=810

\section{Swiss Medical Board}

En janvier 2012, le gouvernement de la Principauté du Liechtenstein s'est associé aux organes responsables du Swiss Medical Board. 